\title{
Características del acoso laboral de los empleados del Sector Comercio en el Municipio de Antiguo Cuscatlán y Sta.Tecla, Departamento de La Libertad, El Salvador
}

Mención Honorífica de Administración de Empresas

Investigacion realizada por: Claudia Carolina Cornejo Escalante, Neri Yudith Fabián Lara,

Wendy Jeannette Gómez Orellana y Katya Gabriela Mejía Montano

\section{Resumen}

En este artículo se presentan los resultados del estudio sobre el acoso laboral en pequeñas empresas del sector comercio de Antiguo Cuscatlán y Santa Tecla el cual se elaboró para optar al grado de Licenciadas en Administración de Empresas de la Universidad Centroamericana José Simeón Cañas (UCA). De acuerdo a la investigación, el tipo de acoso laboral más sobresaliente es el descendente, es decir, agresiones realizadas por jefes. Asimismo, el género con mayor presencia de acoso laboral es el género masculino. Además entre las características que más identifican a las víctimas son: comprometidos con el servicio, sensibles, honrados, empáticos, entre otras. Y las características que definen al agresor son: falta de empatía, envidia, insensibilidad, entre otras.

\section{Palabras Claves}

Acoso laboral, agresiones, víctima, agresor.
Introducción y marco teórico

$\mathrm{n}$ el acoso laboral intervienen dos sujetos: la víctima y el agresor. De acuerdo a Riquelme (2006), la víctima se caracteriza por ser una persona empática, afectiva, sensible, honrada digna de confianza, buen organizadora, entre otras. Mientras que el agresor se considera como una persona con falta de empatía, insensible, irresponsable, envidiosa, entre otras. Adicionalmente, existen diferentes tipos de acoso laboral según la posición jerárquica de la víctima y el agresor.

Este estudio es de importancia, ya que se pretende identificar el nivel de conocimiento de los empleados acerca del acoso laboral, el género más vulnerable a ser víctima de acoso y las características del acoso laboral. Entre las cuales se encuentran tipos de acoso laboral, características de víctimas, características de agresores y las fases del acoso laboral. Para dicha investigación se tomo una muestra de 150 empleados mayores de 18 años, pertenecientes a pequeñas empresas del sector comercio de los municipios de Antiguo Cuscatlán y Santa Tecla.

\section{Definición y características del acoso laboral}

El término de mobbing o acoso laboral se expande a lo largo del tiempo. Por lo que autores, que han realizado investigaciones sobre esta problemática, definen este fenómeno como toda conducta abusiva y maltrato que atenta contra la dignidad de una persona. En la siguiente figura se muestran conceptos sobre el acoso laboral de acuerdo a Heinz Leymann, M.F Hirigoyen e Iñaki Piñuel:
Heinz Leyman: "una persona ejerce violencia extrema de manera recurrente, para destruir sus redes de comunicación".

M.F Hirigoyen: " Conducta agresiva repetitiva que atenta la dignidad, para poner en peligro su empleo o degradar el ambiente"

Iñaki Piñuel: "Continuo maltrato social que recibe un trabajador, para su destrucción y salida de la organización".

Fuente: elaboración propia a partir de (Rojo \& Cervera, 2005) (Peña Pérez, 2014)(Rivas Sánchez, 2003).

A partir de los conceptos anteriores se pudo definir el acoso laboral como comportamientos agresivos y recurrentes que una persona ejerce sobre otra en su lugar de trabajo, para lograr destruir tanto la carrera profesional como personal del individuo.

Las organizaciones presentan características que propician la aparición del acoso laboral como lo son: organizaciones improvisadas con directivos altamente despreocupados, abuso de poder y manipulación perversa por parte de los acosadores, falta de métodos eficaces para la solución de problemas, alta competitividad social y la gestión autoritaria para optimizar resultados (Ovejero Bernal, 2006).

Además, el acoso laboral posee características que ayudan a conocer más sobre el tema, dentro de estas se encuentran: tipos de acoso laboral, perfil de la víctima y agresor, fases del acoso laboral. A continuación detallamos cada una de ellas: 
Tipos de acoso laboral

Los tipos de acoso laboral, de acuerdo a Rojo \& Cervera (2005) se clasifica por posición jerárquica, en la cual dependiendo de la posición jerárquica del acosador y la víctima dentro de la empresa este puede ser mobbing horizontal, en el que uno o más trabajadores del mismo nivel ejercen acoso laboral sobre otros.

Además, dentro de esta clasificación se encuentra el mobbing vertical, en el cual existen dos vías: La primera, es el mobbing ascendente, donde el acoso es ejercido por uno o varios subordinados hacia el superior en la organización. La otra vía es mobbing descendente, el cual se manifiesta cuando el superior ejerce acoso laboral hacia uno o varios subordinados.

\section{Fases del acoso laboral}

Dentro del acoso laboral se identifican diversas fases y de acuerdo a Piñuel (2001) se establecen las siguientes: inicia con la fase de incidentes críticos. Es decir, cuando se da un conflicto común que ocurre en cualquier entorno. Pero si no es solucionado sigue la segunda fase, la cual es de acoso y estigmatización, donde se hostiga e irrita a la víctima con ataques prolongados, por lo que la víctima llega a sentir culpabilidad.

Si esto persiste, se da lugar a la siguiente etapa, la fase de intervención de la dirección, en la cual intervienen los directivos para solucionar el problema. Pero considerando que la principal responsable es la víctima, juzgando las características de la persona acosada y no los factores del entorno.

Después se procede a la fase de solicitud de ayuda especializada externa y diagnóstico incorrecto, en la cual no hay ningún especialista que ayuda a solucionar el problema que se origina en la organización y se le proporciona diagnósticos erróneos a la víctima como estrés, depresión, neurosis, entre otros. Finalmente, se encuentra la fase de salida o exclusión de la organización, donde la persona acosada puede ser despedida por baja productividad, faltas recurrentes, etc. o la víctima decide renunciar por el acoso recibido.

Perfil de los sujetos que intervienen en el acoso laboralDentro del acoso laboral intervienen dos sujetos,
Ios cuales son el acosador y la víctima. De acuerdo a Riquelme (2006), entre las características que describen el perfil del acosador están la inseguridad, falta de empatía, insensible, incapacidad para las relaciones interpersonales, irresponsabilidad, envidia, entre otras. Se destacan los rasgos de sentimiento de superioridad, desprecio hacia los demás y necesidad de admiración.

La víctima por su parte, presenta características como empático, afectivo, sensible, honrado, digno de confianza, buen organizador, entre otras. Son principalmente personas carismáticas y competitivas, en algunos casos se caracterizan por discapacidad física o mental, con carácter depresivo o altamente eficiente.

Consecuencias del acoso laboral

Indudablemente, el acoso laboral ocasiona efectos negativos tanto físicos como mentales y de manera indirecta a la familia y amigos de las víctimas, entre los efectos que menciona Piñuel (2001) se encuentran: Efectos cognitivos e hiperreacción psíquica como olvido o pérdida de memoria, dificultades para concentrarse, decaimiento o depresión, apatía, irritabilidad, nerviosismo, agresividad, sentimientos de inseguridad e hipersensilidad. También se dan síntomas psicosomáticos de estrés como pesadillas, dolores de estómago, colon irritable, vómitos, náuseas, falta de apetito, sensación de nudo en la garganta, llanto y aislamiento.

Entre los síntomas de desajuste del sistema nervioso autónomo están los dolores en el pecho, sudoración, sequedad en la boca, palpitaciones, sofocos, sensación de falta de aire e hipertensión. También se dan síntomas de desgaste físico producido por un estrés mantenido durante mucho tiempo, trastornos del sueño, cansancio y debilidad.

\section{Aspectos legales y experiencias} regulatorias del acoso laboral

Un aspecto que se desea destacar, es que en El Salvador el acoso laboral es un problema que sufren los empleados y no existe un marco legal que los respalde ante dicha situación. El país no cuenta con leyes específicas para la prevención y atención de las personas víctimas de acoso laboral, pero podemos tomar en cuenta las leyes para la protección de los derechos humanos, entre ellas se encuentran:

\section{Leyes y códigos que respaldan a las víctimas del acoso laboral}

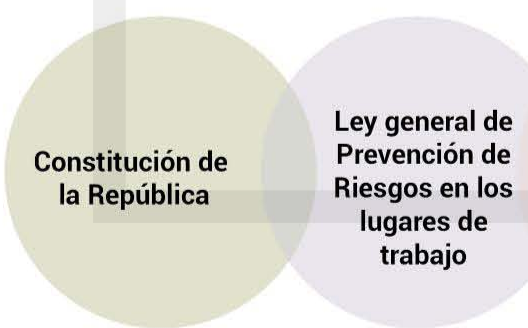
Ley de igualdad, equidad y
Erradicación de la
Discriminación contra las mujeres

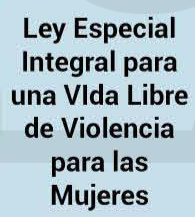

Fuente: Elaboración 'propia a partir de información de Secretaría para Asuntos Estratégicos de la Presidencia(2013) 
Estas leyes y códigos, que se pueden considerar como respaldo legal del acoso laboral, no mencionan explícitamente el término, sino que incluyen manifestaciones del mismo, como: daños psicológicos, violencia de género y discriminación (Secretaría para Asuntos Estratégicos de la Presidencia, 2013).

\section{Metodología}

Para la realización de esta investigación se creo un instrumento, el cual denominamos Instrumento de Medición ALESTA (Instrumento de Medición de Acoso Laboral en Santa Tecla y Antiguo Cuscatlán). Para su elaboración se consultaron herramientas que permiten la medición del acoso laboral. En primer lugar se reviso la Escala Cisneros' ${ }^{1}$, luego se examino cada uno de los comportamientos del instrumento de Leymann ${ }^{2}$, para lograr complementar el instrumento con conductas de hostigamiento que no posee la primera escala y que son relevantes para la investigación, asimismo en las agresiones realizamos adaptaciones al lenguaje de El Salvador.

El cuestionario está constituido en dos partes: en la primera parte, se formularon preguntas para determinar el nivel de conocimiento sobre el acoso laboral; en la segunda parte, se detallan una serie de agresiones para identificar si las personas han sido víctimas de acoso. Seguidamente de otras preguntas para conocer las características, fases y otros aspectos del acoso laboral. El universo sobre el que se realizo el estudio es el personal ocupado de los municipios de Santa Tecla y Antiguo Cuscatlán. La muestra está constituida por 150 empleados mayores de 18 años, pertenecientes a pequeñas empresas del sector comercio. Dicha muestra está caracterizada por 76 personas del género masculino, de los cuales 36 hombres pertenecen a Santa Tecla y 40 a Antiguo Cuscatlán; 74 personas del género femenino, de las cuales 35 pertenecen a Santa Tecla y 39 a Antiguo Cuscatlán. El procedimiento que se utilizo para recolectar la información fue la visita de pequeñas empresas del sector comercio, lugares públicos y la UCA, en donde realizamos entrevistas a empleados. Asimismo, se envio el cuestionario vía correo electrónico.

\section{Resultados y discusión}

\section{Nivel de conocimiento}

El acoso laboral es un término que está presente en la mayoría de las empresas, por ello en el desarrollo del estudio se les consulto a las personas si conocían la definición del acoso laboral. De 116 empleados que entrevistamos, y que manifiestan tener conocimiento sobre el acoso laboral, el $81 \%$ conoce con total claridad sobre el tema, mientras que el $19 \%$ realmente no lo conoce. Por lo que, de acuerdo a los resultados, se procede a crear una nueva variable para clasificar a las personas que realmente conocen el término y las personas que no, de esta manera se obtuvo que, de la muestra total de 150 empleados, un $37.3 \%$ no sabe en qué consiste el término 3 del acoso laboral y un $62.7 \%$ sí conoce dicho término. Los empleados conceptualizan el acoso laboral como hostigamiento, maltrato, molestias, abuso de poder, sobrecarga laboral, amenazas, agresiones, insinuaciones, violaciones, denigración de la dignidad humana, etc. A continuación, se muestran los porcentajes para cada una de las definiciones antes mencionadas:

\section{Definiciones de acoso laboral}

\begin{tabular}{l|c|c|}
$\begin{array}{l}\text { Hostigamiento, maltrato y/o } \\
\text { molestia en el trabajo. }\end{array}$ & 31 & $26.7 \%$ \\
\hline $\begin{array}{l}\text { Abuso de poder, sobre carga } \\
\text { laboral. }\end{array}$ & 21 & $18.1 \%$ \\
\hline $\begin{array}{l}\text { Amenazas, agresiones o acciones } \\
\text { que ponen en riesgo a la persona. }\end{array}$ & 13 & $11.2 \%$ \\
\hline $\begin{array}{l}\text { Acoso psicológico, verbal o físico } \\
\text { Actitudes inapropiadas, falta de }\end{array}$ & 13 & $11.2 \%$ \\
\hline $\begin{array}{l}\text { respeto, insinuaciones } \\
\text { Denigrar la dignidad humana y }\end{array}$ & 10 & $8.6 \%$ \\
\hline $\begin{array}{l}\text { violación de derechos } \\
\text { Forzar a tomar decisiones } \\
\text { involuntariamente }\end{array}$ & 1 & $0.9 \%$ \\
\hline $\begin{array}{l}\text { Definición inprecisa } \\
\text { Total }\end{array}$ & 22 & $116.0 \%$ \\
\hline
\end{tabular}

Fuente: Elaboración propia a partir de datos de la investigación.

Por otra parte, por medio del Instrumento de Medición ALESTA se identifican a los empleados que estaban siendo víctimas de acoso laboral, y los resultados indican que un $28.7 \%$ (43 personas) manifiesta ser víctima de acoso laboral.

De las 43 personas que manifiestan ser víctimas de acoso laboral, el $69.8 \%$ confirma tener claro el concepto de acoso laboral y el $30.2 \%$ no lo tiene claro, se logró evidenciarlo porque tienden a asociarlo directamente con el abuso sexual y con la presión o exigencia en el cumplimiento de sus obligaciones laborales, las cuales son definiciones imprecisas del término.

Además el $23.3 \%$ de las 43 personas que han sido víctimas de acoso laboral, no están consciente de ello.

*1 La escala Cisneros está constituida por 43 preguntas, las cuales se responden de acuerdo al grado de frecuencia que se producen las conductas de hostigamiento en las personas, para esto se encuentra definido una escala que va desde 0 a 6. donde cero significa "nunca" y seis "todos los días". *2 El Leymann Inventory of Psychological Terrorization (LIPT) es un instrumento diseñado por Leymann, está estructurado por medio de 60 preguntas, las cuales se responden a través de una valoración escalar para cada una de las conductas de acoso; la cual va "desde cero (la conducta no ha tenido lugar) hasta cuatro (conducta de intensidad máxima)".

$* 3$ En la nueva variable creada, las personas que no conocen el término de acoso laboral son aquellas que definitivamente indicaron que no lo conocían más las personas que poseen una definición imprecisa sobre éste. 


\section{Acoso laboral ejercido según género}

El acoso laboral puede variar de un género a otro, debido a que los hombres pueden sentirse mucho más identificados con ciertas agresiones en comparación con las mujeres o viceversa.
Los resultados de este estudio reflejan que de 74 mujeres, el $23 \%$ ha sido víctima de maltrato en los últimos seis meses. Mientras que de 76 empleados del género masculino, un $34.2 \%$ ha sido víctima de acoso laboral, tal y como mostramos en la siguiente tabla:

\section{Acoso laboral por género}

\begin{tabular}{|c|c|c|c|c|c|c|}
\hline \multirow{3}{*}{ Género } & \multicolumn{6}{|c|}{ Ha sido víctima de acoso laboral en el transcurso de 6 meses } \\
\hline & \multicolumn{2}{|c|}{ SI } & \multicolumn{2}{|c|}{ NO } & \multicolumn{2}{|c|}{ TOTAL } \\
\hline & $\mathrm{Fr}$ & $23.0 \%$ & $\mathrm{Fr}$ & $\%$ & $\mathrm{Fr}$ & $\%$ \\
\hline Femenino & 17 & $\%$ & 57 & $77.0 \%$ & 74 & $49.3 \%$ \\
\hline Masculino & 26 & $34.2 \%$ & 50 & $65.8 \%$ & 76 & $50.7 \%$ \\
\hline $\begin{array}{l}\text { ente: Elabo } \\
\text { la investig }\end{array}$ & & $r d e$ & & & 150 & $100.0 \%$ \\
\hline
\end{tabular}

De acuerdo al estudio denominado: "La violencia en el trabajo: un problema mundial", realizado por la Organización Internacional del Trabajo (1998), afirma que las mujeres son las que tienen más riesgo de ser víctimas de acoso debido a que están expuestas a ocupaciones como el comercio minorista, la enseñanza, el trabajo social, la enfermería y la banca. Pero en El Salvador, de acuerdo a estudios realizados por la Organización de Mujeres Salvadoreñas por la Paz (2009), de 82 casos que han sufrido de agresión física y maltrato; 40 de ellas son mujeres y los otros 42 casos son hombres. Además, con nuestro estudio confirmamos que los hombres son más agredidos que las mujeres.

Por otra parte, de la muestra total de 150 personas, 76 fueron hombres, los cuales presentan los siguientes porcentajes de acoso 4

En promedio, un $86.7 \%$ manifiesta que no ha sufrido acoso laboral, un $6.4 \%$ afirma haber sufrido agresiones con poca frecuencia, un $3 \%$ opina que ha sufrido de maltratos con una frecuencia moderada, mientras que un $2.4 \%$ señala que con mucha frecuencia y el $1.4 \%$ manifiesta recibir agresiones de manera extrema.

\section{Tipos y características de acoso laboral}

\section{Tipos de acoso según la posición jerárquica}

De acuerdo a los resultados y al promedio de frecuencia de agresión por parte de los jefes, compañeros de trabajo y colaboradores, existen los siguientes tipos de acoso:

Acoso laboral descendente: De acuerdo a la escala de frecuencia de las agresiones presentadas en el instrumento de investigación, en promedio un $21 \%$ de las personas entrevistadas manifiestan que han presentado acoso por parte de los jefes. Estos distribuidos en frecuencias de poco, moderadamente, mucho y extremadamente, tal y como se muestra en el siguiente gráfico:
Media del Acoso laboral descendente

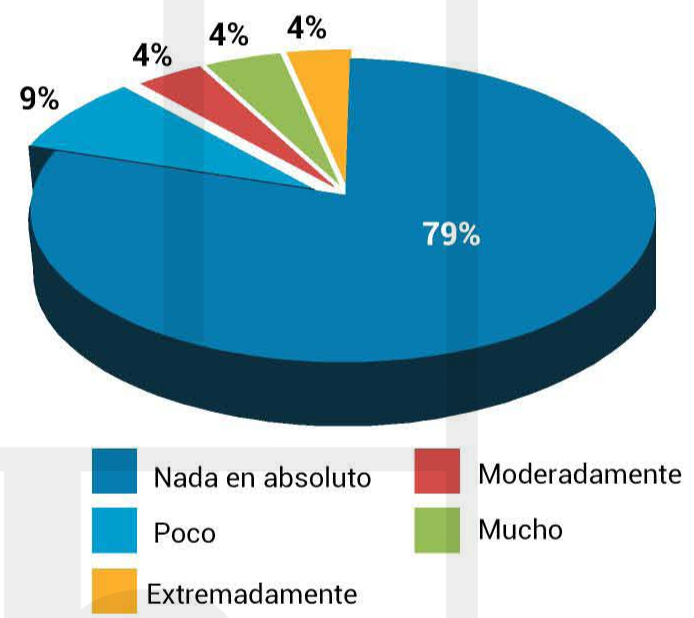

Fuente: Elaboración propia a partir de datos de la investigación.

Para cada una de las agresiones se realizó una media, en donde se muestra cuál de éstas se realiza con mayor frecuencia por parte de los jefes. Entre las agresiones que se dan con mayor y menor frecuencia para este tipo de acoso se encuentran:

- Me controlan de manera muy estricta mi horario, con una media de 1.1, lo cual significa que esta agresión tiende más a poco que a modernamente.

- Me ocasionan daños en mis pertenencias o en mi vehículo, con una media de 0.1 , lo cual indica que esta agresión tiende a una frecuencia de nada en absoluto.

- Me empujan físicamente para intimidarme, con una media de 0.1 , lo cual indica que esta agresión tiende a una frecuencia de nada en absoluto. 
Es importante aclarar, que la agresión "me controlan de manera muy estricta mi horario" es una manifestación de acoso laboral, la cual era de total desconocimiento por las personas, pero con el Instrumento de Medición ALESTA lograron identificar dicha agresión como acoso laboral. Tomando como base las agresiones antes mencionadas realizamos cruces de variables, donde se muestra la frecuencia con que se realizan estas agresiones, en las cuales:

- Diecinueve personas señalan que con una frecuencia extrema, sus jefes controlan de manera estricta su horario y no los empujan físicamente para intimidarlos.

- Una persona manifiesta que de forma extrema, su jefe controla de manera estricta su horario, y con una frecuencia moderada la empuja físicamente para intimidarla.

- Dos personas señalan que no sufren de daños en sus pertenencias o en su vehículo, pero con una frecuencia moderada sus jefes los empujan físicamente para intimidarlos.

- Una persona indica que con poca frecuencia, su jefe le ocasiona daños a sus pertenencias o en su vehículo, y con mucha frecuencia su jefe la empuja físicamente para intimidarla.

Acoso laboral horizontal: De acuerdo a la escala de frecuencia de las agresiones presentadas en el instrumento de investigación y al siguiente gráfico, en promedio un $11.3 \%$ de los empleados son acosados por sus compañeros de trabajo. Estas agresiones se dan con frecuencia de poco, moderadamente, mucho y extremadamente.

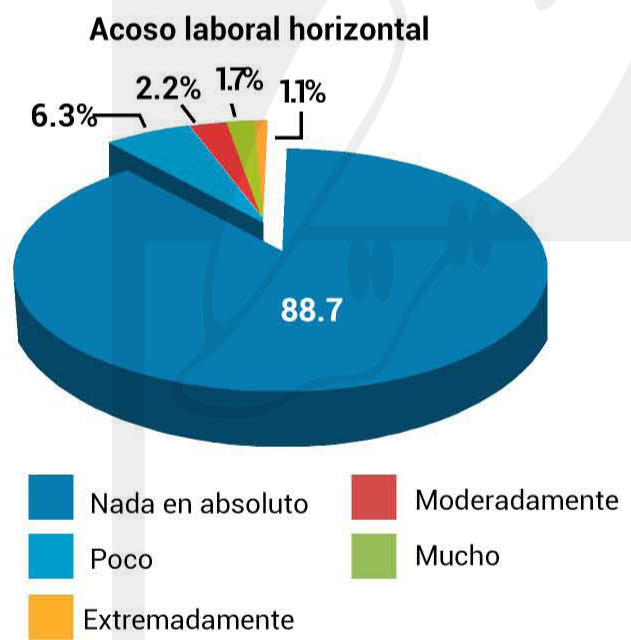

Fuente: Elaboración propia a partir de datos de la investigación.

Para cada una de las agresiones se realizó una media, en la cual se muestra cuál de estas se realiza con mayor frecuencia por los compañeros de trabajo. Entre las agresiones que se dan mayor y menor frecuencia para este tipo de acoso se encuentran:

- "Me cortan mis iniciativas, no me permiten desarrollar mis ideas", con una media de 0.4 , lo cual indica que esta agresión tiende más a una frecuencia de nada en absoluto que a poco.

- "Me hacen burla o bromas intentando ridiculizarme", con una media de 0.4 , lo cual indica que esta agresión tiende más a una frecuencia de nada en absoluto que a poco.

- "Cuando solicito permiso me lo niegan o me ponen dificultades", con una media de 0.05 , lo cual indica que esta agresión tiende a una frecuencia de nada en absoluto.

Es importante aclarar, que las agresiones "me cortan mis iniciativas, no me permiten desarrollar mis ideas" y "cuando solicito permiso me lo niegan o me ponen dificultades" son manifestaciones de acoso laboral, las cuales eran de total desconocimiento por las personas, pero con el Instrumento de Medición ALESTA lograron identificar dichas agresiones como acoso laboral.

De las agresiones antes mencionadas se realizaron cruces de variables, donde se muestra la frecuencia con que se realizan estas agresiones, en las cuales:

- Dos empleados manifestaron que de una forma extrema sufren de burlas o bromas intentando ridiculizarlos, y con mucha frecuencia les cortan sus iniciativas.

- Dos personas han sufrido con poca frecuencia de burlas o bromas por parte de sus compañeros y con igual frecuencia les niegan permisos cuando lo solicitan.

- Seis empleados manifiestan que sufren de manera extrema de burlas y bromas, pero no les niegan los permisos cuando los solicitan.

Acoso laboral ascendente: De acuerdo a la escala de frecuencia de las agresiones presentadas en el instrumento de investigación, en promedio un $5 \%$ manifiesta sufrir de agresiones por parte de los colaboradores, con frecuencia de poco, mucho, moderadamente y extremadamente, tal y como se refleja a continuación:

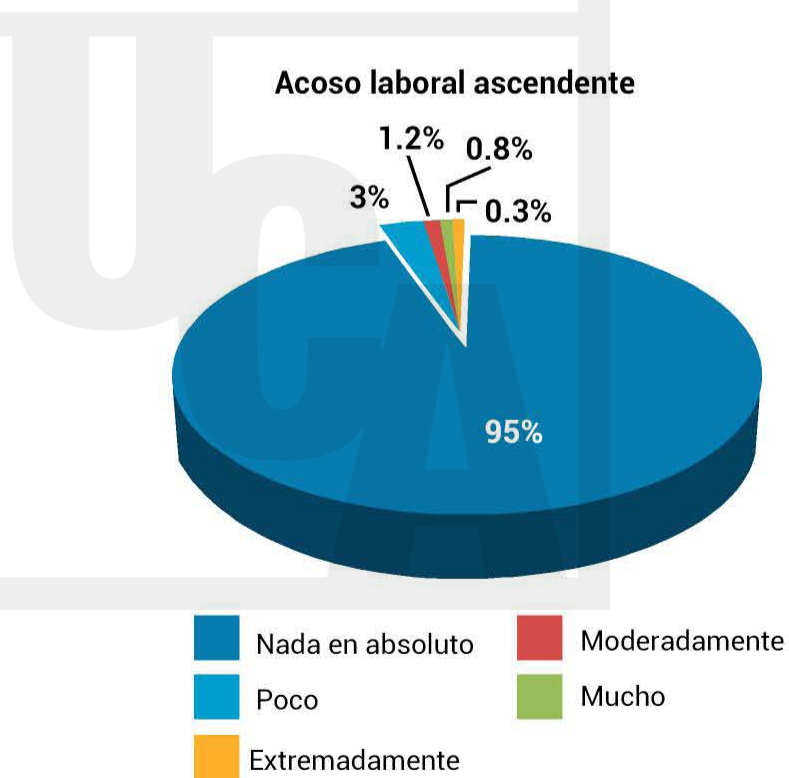

Fuente: Elaboración propia a partir de datos de la investigación. 
Para cada una de las agresiones se realizó una media, en donde se muestra cuál de estas se realiza con mayor frecuencia por los colaboradores. Entre las agresiones que se dan con mayor y menor frecuencia en este tipo de acoso se encuentran:

- "Recibo amenazas verbales y escritas o gestos intimidantes", con una media de 0.2 , lo cual indica que esta agresión tiende a una frecuencia de nada en absoluto.

- "Me controlan de manera muy estricta mi horario", con una media de 0.2 , lo cual indica que esta agresión tiende a una frecuencia de nada en absoluto.

- "Recibo una presión indebida para sacar adelante mi trabajo", con una media de 0.2 , lo cual indica que esta agresión tiende a una frecuencia de nada en absoluto.

- "Me empujan físicamente para intimidarme", con una media de 0.01 , lo cual indica que esta agresión tiende a una frecuencia de nada en absoluto.

- "Utilizan varias formas de hacerme incurrir en errores profesionales de manera malintencionada", con una media de 0.01 , lo cual indica que esta agresión tiende a una frecuencia de nada en absoluto.

Es importante aclarar que las agresiones "me controlan de manera muy estricta mi horario" y "utilizan varias formas de hacerme incurrir en errores profesionales de manera malintencionada" son manifestaciones de acoso laboral, las cuales eran de total desconocimiento por las personas, pero con el Instrumento de Medición ALESTA lograron identificar dichas agresiones como acoso laboral. De las agresiones antes mencionadas realizamos cruces de variables, donde se muestra la frecuencia con que se realizan estas agresiones, en las cuales:

- Dos empleados manifiestan que con mucha frecuencia, sus colaboradores controlan de manera muy estricta su horario y reciben una presión indebida para sacar adelante su trabajo.

- Una persona indica que con una frecuencia extrema, sus colaboradores le controlan de manera muy estricta su horario y recibe una presión indebida para sacar adelante su trabajo.

- Una persona señala que con poca frecuencia le controlan de manera estricta su horario y utilizan varias formas de hacerle incurrir en errores profesionales de manera malintencionada.

Por lo tanto, de acuerdo a los tipos de acoso laboral podemos determinar que el tipo de acoso más sobresaliente es el descendente, seguidamente del horizontal y por último el ascendente.

\section{Características de los acosadores y las víctimas}

De acuerdo a los resultados del estudio, se confirma que las principales características asociadas a los agresores son: falta de empatía con un $54 \%$, seguidamente de la envidia y la insensibilidad con un $44.7 \%$ y un $25.3 \%$, respectivamente.

Entre otras características más relevantes indicadas por los empleados son la mentira compulsiva y la irresponsabilidad, con $18.7 \%$ y $18 \%$, respectivamente. Adicionalmente, los resultados muestran que un $44.7 \%$ de las opiniones de los empleados de las empresas del sector comercio, manifiestan que las víctimas son personas comprometidas con el servicio, un $44 \%$ manifiesta que son sensibles, un $27.3 \%$ opina que son honrados. El $23.3 \%$ opina que se caracterizan por ser empáticas y un
$15.3 \%$ indica que son buenos organizadores. Al analizar la información de forma muy arriesgada, podemos decir que los agresores por la inseguridad a perder su trabajo o perder la admiración que poseen, tienden agredir a las personas sobresalientes para que éstos se sientan hostigados y abandonen su trabajo. Asimismo, por la satisfacción que les genera denigrar a otras personas. Fases del acoso laboral

En el instrumento de investigación, se formuló una pregunta para conocer la fase en la que se encuentran los empleados entrevistados. A continuación se muestran los resultados obtenidos sobre dicha pregunta:

Un $57.3 \%$ de los empleados, manifiesta encontrarse en la primera fase del acoso laboral. Es decir, presentan leves conflictos por diferencia de opiniones, actitudes o roces. Por lo que estos problemas pueden llegar a solucionarse o convertirse en un problema de mayor magnitud. El 10.7\% manifestó que ha pensado abandonar su trabajo por las agresiones recibidas, y un $3.3 \%$ ha presentado agresiones y ha buscado ayuda especializada.

Otros aspectos del acoso laboral

Otro apartado, el cual se discutirá, será la edad más frecuente en la cual las personas sufren agresiones y las políticas de acoso laboral de las pequeñas empresas del sector comercio en los municipios de Santa Tecla y Antiguo Cuscatlán.

De las 43 personas que sufren de acoso en su lugar de trabajo, un $18.6 \%$ se encuentra entre las edades de 18 a 22 años; un $32.6 \%$ se encuentra entre las edades de 23 a 27 años, siendo personas que probablemente recientemente han ingresado al mercado laboral y no poseen otra alternativa para continuar con su desarrollo profesional. Seguidamente, se encontró que un $27.9 \%$ se encuentra entre 33 a más años de edad, ya que por ser personas mayores pueden tener ciertas dificultades, como el manejo de la tecnología o el desarrollo de algunas actividades, por lo que pueden ser denigradas por personas con una mayor preparación profesional.

Por otra parte, los resultados de la investigación demuestran que un $31.3 \%$ de los empleados entrevistados, manifiestan que las empresas en las cuales trabajan no poseen políticas para prevenir el acoso laboral y un $30 \%$ indica que no sabe, tal y como se refleja a continuación:

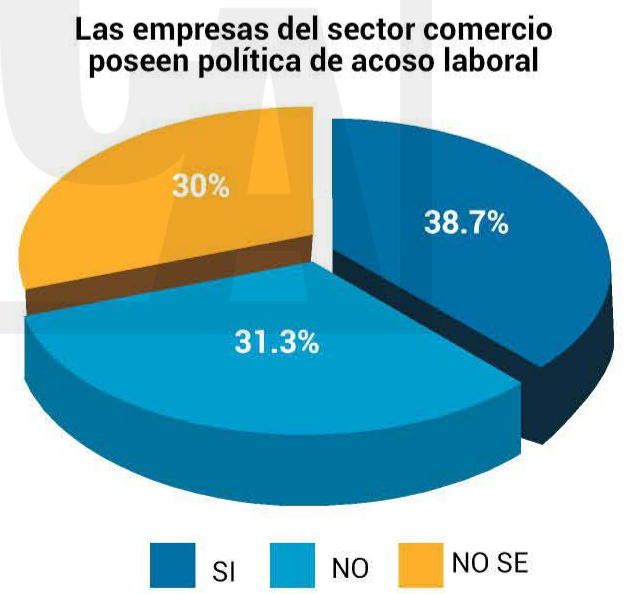

Fuente: Elaboración propia a partir de datos de la investigación. 
Un $37.9 \%$ de los empleados que manifiestan que en la empresa en la cual laboran poseen política de acoso laboral, no saben a qué instancia recurrir dentro de la empresa en caso de ser víctima de acoso. Por tanto, se puede deducir que las empresas no le dan importancia necesaria al tema del acoso laboral.

\section{Conclusiones}

En este apartado se abordan tres ejes: empresarios, empleados e instituciones formadoras, los cuales son generadores de cambio para una productividad mayor y un desarrollo integral de la persona-empleado dentro de la empresa.

El primer eje es el de los empresarios, debido que dentro de una empresa el recurso más valioso es el recurso humano y se necesita tanto de ellos como de otros recursos para ser productivos. Por lo tanto estas deben cuidar del personal mediante: una selección adecuada; crear y adoptar políticas preventivas, contra conductas de acoso laboral; asimismo, informar sobre el tema del acoso laboral por medio de boletines informativos.

Además, las empresas deben contemplar el tema del acoso laboral, ya que al no tratarse adecuadamente puede generar efectos como: abandono de trabajo, baja productividad, demandas hacia la empresa, daños hacia las pertenencias de la empresa o del personal, entre otros.

El segundo eje es el de los empleados, ya que el clima laboral tiene un impacto grande en el desarrollo del empleado. Por lo tanto, cuando este es víctima de acoso laboral se le recomienda: tener claro el término de acoso laboral, no tener temor a expresar sus pensamientos y emociones, no ser testigo, e informar a las autoridades internas correspondientes. En caso que el empleado no tenga respuesta interna. Es decir, por parte de empresa para resolver amenazas de acoso laboral, debe recurrir a entidades gubernamentales como la Procuraduría de la Defensa de los Derechos Humanos, Ministerio de trabajo, entre otros.

El tercer eje es el involucramiento de instituciones formadoras. Entre estas se encuentran: las instituciones de educación media, las cuales deben informar sobre el acoso laboral, para que las personas tengan un conocimiento y una preparación más sólida al ingresar al ámbito laboral o al enfrentar esta situación.

Además, se encuentran las instituciones de educación superior, a las cuales se les recomienda impartir esta problemática como materia y realizar charlas informativas del acoso laboral, gratuitas y abiertas al público en general. Esto para multiplicar el conocimiento del acoso laboral, sus causas y efectos. Asimismo, el Gobierno debe crear una ley específica del acoso laboral para que la población activa ocupada tenga esa información y sea respaldada por esa ley. Adicionalmente, debe crear grupos de apoyo en los cuales las víctimas de acoso laboral puedan recibir un tratamiento necesario según su grado de afección.

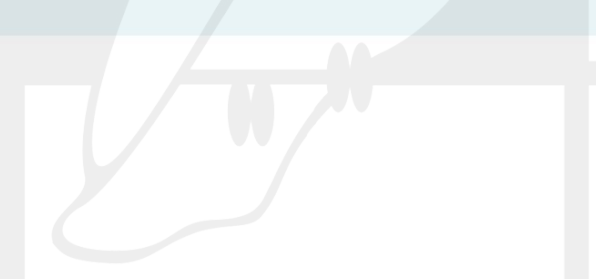

Bibliografía:

Organización Internacional del Trabajo. (1998). La violencia en el trabajo: un problema mundial. Recuperado el 8 de abril de 2016, de http://www.ilo.org/global/about-the-ilo/newsroom/news/WCMS_008502/lang--es/index.htm

Organización de Mujeres Salvadoreñas por la Paz. (2009). Estudio sobre el Conocimiento de los Derechos Laborales en El Salvador, 2009. Obtenido de

http://observatoriolaboral.ormusa.org/investigaciones/Estudio_sobre_conocimiento_de_Derechos_Laborales_en_ El_Salvador.pdf

Ovejero Bernal, A. (2006). El mobbing o acoso psicológico en el trabajo: una perspectiva psicosocial. Recuperado el 24 de Abril de 2016, de http://www.redalyc.org/articulo.oa?id=231317045005

Peña Perez, R., \& Colaboradores. (2014). Cómo enfrentarse al acoso laboral o mobbing. Guía personal y profesional. España: Publicaciones Altaria.

Piñuel, I. (2001). Mobbing: cómo sobrevivir al acoso psicológico en el trabajo (1 ed.). Cantabria: Sal Terrae.

Riquelme, A. (2006). Mobbing, un tipo de violencia en el lugar de trabajo. Ciencias Sociales Online, III(2). 